\title{
AN OBSERVATIONAL STUDY ON ANTI-THYROPEROXIDASE ANTIBODY POSITIVITY IN THYROIDITIS CASES AMONG ADULTS IN A TERTIARY CARE CENTER
}

\author{
Aneesha Fathima T. S1, Y. Shajahan² \\ ${ }^{1}$ Senior Resident, Department of General Surgery, Government TD Medical College, Alappuzha, Kerala, India. \\ ${ }^{2}$ Additional Professor, Department of General Surgery, Government TD Medical College, Alappuzha, Kerala, India.
}

\begin{abstract}
BACKGROUND
ABSTRACT

The major cause of hypothyroidism in adults is Hashimoto thyroiditis. Thyroperoxidase (TPO) is an enzyme involved in thyroid hormone synthesis. And anti-TPO antibodies are raised in cases of Hashimoto's thyroiditis. We wanted to study the incidence of anti-TPO antibody positivity in thyroiditis cases.
\end{abstract}

\section{MATERIALS AND METHODS}

All the patients attending surgery OPD from Jan. 2016 to Dec. 2016 who were FNAC proven cases of Hashimoto thyroiditis were selected and sent for anti-TPO antibody positivity and the proportion of patients with positive result were compared according to their thyroid functional status using various statistical methods.

\section{RESULTS}

It was an observational study and the idea was to see the accuracy with which the FNAC finding of thyroiditis correlated with antiTPO antibody positivity. And we also looked for thyroid function status of all the patients. Comparison was done of each group in relation to thyroiditis and anti-TPO antibody positivity. 97\% thyroiditis cases were positive for anti-TPO antibody.

\section{CONCLUSIONS}

The test for anti-TPO antibody positivity can be used to detect cases of Hashimoto's thyroiditis with $97 \%$ certainty. The anti-TPO antibody testing can be used along with regular testing of TFT, FNAC in cases of thyroiditis.

HOW TO CITE THIS ARTICLE: Fathima ATS, Shajahan Y. An observational study on anti-thyroperoxidase antibody positivity in thyroiditis cases among adults in a tertiary care center. J. Evolution Med. Dent. Sci. 2019;8(13):1003-1008, DOI: $10.14260 /$ jemds/2019/223

\section{BACKGROUND}

The major cause of hypothyroidism in adults is Hashimoto's thyroiditis an autoimmune-mediated destruction of thyrocytes. A complex immunologic phenomenon results in the formation of immune complexes and complement in the basement membrane of follicular cells. Alterations in thyroid cell function result that impair T3 and T4 production. These cellular reactions ultimately result in the infiltration of lymphocytes and resultant fibrosis, which decreases the number and efficiency of individual follicles. As this immune phenomenon continues, the presence of TSH-blocking antibodies, such as thyroid peroxidase antibodies, can be detected. Thyroid peroxidase antibodies are probable key mediators in the initial complement fixation process. As the immune process continues, thyroid function can be altered by levels of these antibodies. Ultimately, a clinical hypothyroid state can occur in patients with persistent TSH-blocking antibodies.

Thyroperoxidase (TPO) is an enzyme involved in thyroid hormone synthesis, catalysing the oxidation of iodide on tyrosine residues in thyroglobulin for the synthesis of triiodothyronine and thyroxine (Tetraiodothyronine). ${ }^{1,2}$

'Financial or Other Competing Interest': None.

Submission 08-02-2019, Peer Review 14-03-2019,

Acceptance 20-03-2019, Published 01-04-2019.

Corresponding Author:

Y. Shajahan,

Shamjana, Chirakadavom,

South of Government Hospital, Kayamkulam,

Kerala, India.

E-mail: dr.yshajahan@yahoo.com

DOI: $10.14260 /$ jemds $/ 2019 / 223$

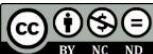

TPO is a membrane-associated hemoglycoprotein expressed only in thyrocytes and is one of the most important thyroid gland antigens.

Disorders of the thyroid gland are frequently caused by autoimmune mechanisms with the production of autoantibodies. Anti-TPO antibodies activate complement and are thought to be significantly involved in thyroid dysfunction and the pathogenesis of hypothyroidism.

\section{Thyroid Autoantibody Levels}

Thyroid antigens (Thyroid-stimulating immunoglobulin, antimicrosomal and antithyroid peroxidase antibodies) are produced in autoimmune thyroid disorders, including Graves" disease and Hashimoto thyroiditis. Detection of autoantibodies can be extremely important if either of these autoimmune conditions is suspected. Approximately 95\% of patients with Hashimoto thyroiditis and $80 \%$ with Graves' disease have detectable anti-microsomal antibodies. In Graves' disease, circulating antibodies have a high affinity for TSH-R on thyroid follicular cells. ${ }^{3}$ Assays have greater sensitivity and may allow earlier detection of Graves' disease and more accurate monitoring of the effects of thyroid medication. Thyroperoxidase (TPO) is an enzyme involved in thyroid hormone synthesis, catalysing the oxidation of iodide on tyrosine residues in thyroglobulin for the synthesis of triiodothyronine and thyroxine (Tetraiodothyronine). Reference Values $<9.0 \mathrm{IU} / \mathrm{mL}$

Reference values apply to all ages. Values above 9.0 $\mathrm{IU} / \mathrm{mL}$ generally are associated with autoimmune thyroiditis, but elevations are also seen in other autoimmune diseases.

The frequency of detectable anti-TPO observed in nonimmune thyroid disease is similar to the $10 \%$ to $12 \%$ observed in a healthy population with normal thyroid 
function. ${ }^{4}$ Hashimoto's thyroiditis (HT) is an autoimmune disease and it is more prevalent in Asians. The incidence of HT seems to be increasing in the recent times. It is one of the most common cause of hypothyroidism. The study was conducted in surgery OPD in T D Medical College Alappuzha for one-year period from January 2016 to December 2016.

\section{Aim}

The purpose of this study was to estimate proportion of antithyroperoxidase antibody positivity among thyroiditis cases in adults in tertiary care centre.

\section{Objectives}

\section{- Primary Objectives}

- To estimate proportion of anti-thyroperoxidase antibody positivity among thyroiditis cases in adults.

\section{- Secondary Objectives}

- To find out association between hypothyroidism and anti-TPO positivity.

\section{MATERIALS AND METHODS}

\section{Study Design}

Cross sectional study.

\section{Study Setting}

Department of Surgery

Government Medical College, Alappuzha.

\section{Study Period}

One year from the date of obtaining ethical clearance. January 2016 - December 2016(12 months)

\section{Study Population}

FNAC proven thyroiditis cases among adult population coming to surgery OP at TDMC Vandanam (age $>18 y$ rs).

\section{Sample Size}

60.

All patients attending surgery OPD fulfilling the inclusion criteria. FNAC confirmed thyroiditis cases are about five to six per month. So, my sample size is about 60 in a year.

\section{Inclusion Criteria}

- Adult FNAC proven thyroiditis cases.

- Those who are willing to give written informed consent

\section{Exclusion Criteria}

- Graves' with anti-TPO antibody positive cases.

- Other diseases with anti-TPO antibody positivity like Type $1 \mathrm{DM}$, connective tissue disorders.

\section{Ethical Clearance}

Ethical clearance was obtained from The Human Ethics Committee T D Medical College, Alappuzha. A written consent was obtained from all the patients included in the study.

\section{Study Procedure}

Patients coming to Surgery department with symptoms of any throid disorder were included. After clinical assessment they are sent for investigations like Ultrasound of thyroid, FNAC thyroid, and blood tests like TSH, free T3, free T4.

Among them those who were having FNAC finding of Hashimoto's thyroiditis were selected as the sample pool. 5

Patients were informed about the study and its objective and after getting a written informed consent, data was collected in proforma.

The FNAC proven Hashimoto's thyroiditis cases were sent for Anti-TPO antibody testing. The test used to detect antiTPO antibody was microplate chemiluminescence immunoassay. The anti-TPO positive or negative irrespective all cases were segregated into hypothyroid, hyperthyroid and euthyroid.

\section{Statistical Analysis}

Analysis was done using Microsoft excel and SPSS. The correlation between anti-TPO positive and hypothyroid cases and other alternate thyroid function was found out using chi square test.

\section{RESULTS}

\section{Observation and Analysis}

The study was conducted in General surgery OPD of T D Medical College, Alappuzha. From time period of January 2016 to December 2016. The study was conducted on total of 60 patients who were above 18yrs and were having thyroid FNAC as Hashimoto thyroiditis.

All of the patients were thoroughly investigated with thyroid function test including serum $\mathrm{TSH}$, free T3 and freeT4. Later all underwent ultrasound scan of thyroid gland and FNAC. All the laboratory parameters were recorded.

Later all the patients who showed Hashimoto thyroiditis were selected and they were subjected to anti-TPO antibody testing which was done using immunoassay (CLIA). The antiTPO antibody test results were collected.

\section{Demographic Profile/Age Distribution}

The age range of patients included in the study is between 18 and 70 years. Table 1 gives the percentage distribution of the thyroiditis cases according to age. The highest distribution was seen in the 4 th and 5 th decade. Around $60 \%$ of patients belonging to this age group. The previous studies regarding prevalence also give the same results as given here.

\begin{tabular}{|c|c|c|}
\hline Age & Count & Percent \\
\hline $21-30$ & 9 & 15.0 \\
\hline $31-40$ & 18 & 30.0 \\
\hline $41-50$ & 19 & 31.7 \\
\hline $51-60$ & 8 & 13.3 \\
\hline $61-70$ & 6 & 10.0 \\
\hline Mean \pm SD & $43.4 \pm 11.9$ \\
\hline Table 1. Percentage Distribution of Thyroiditis Cases \\
According to Age \\
\hline
\end{tabular}




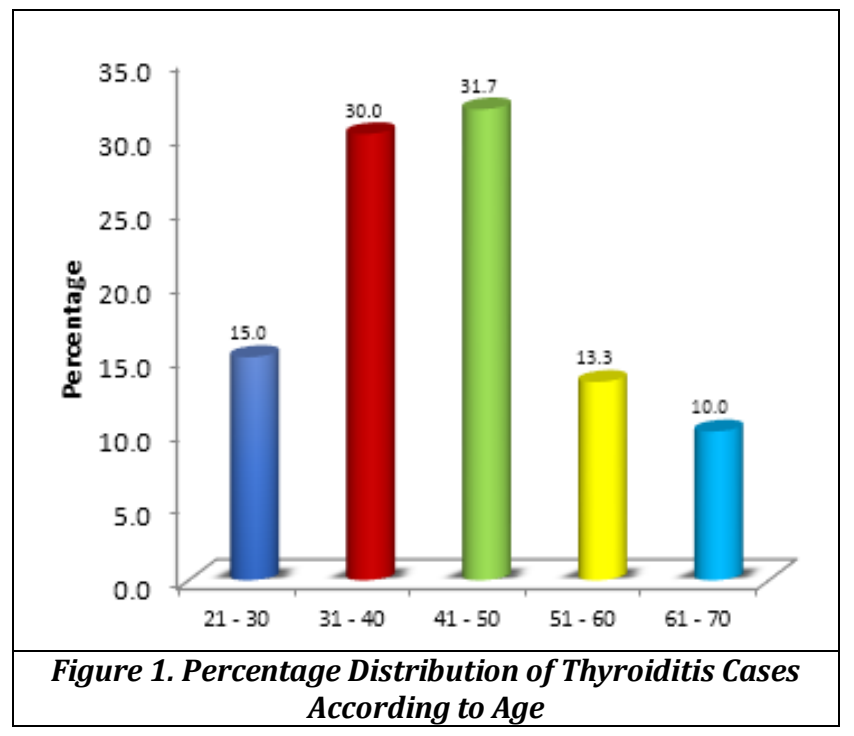

Percentage Distribution of Thyroiditis Cases According to Sex

Thyroid disorders are commonly seen in females than male hence the same with thyroiditis. The percentage according this study is about $83 \%$ which is lesser compared to previous studies. Hence incidence in males have gone up.

\begin{tabular}{|c|c|c|}
\hline Sex & Count & Percent \\
\hline Male & 10 & 16.7 \\
\hline Female & 50 & 83.3 \\
\hline Table 2. Percentage Distribution of the Thyroiditis Cases \\
According to Sex \\
\hline
\end{tabular}

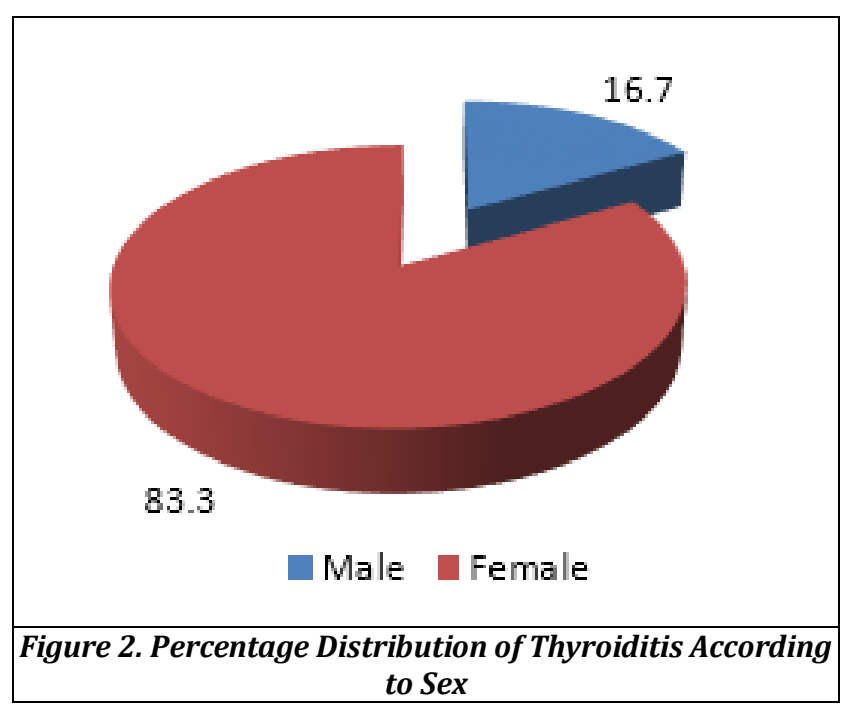

Percentage of Anti-TPO Positivity in Thyroiditis Cases Now, coming to the primary objective of the study, the percentage of anti-TPO positivity in thyroiditis cases. Here there was $96.7 \%$ anti-TPO antibody positivity in FNAC proven thyroiditis cases. Which is similar to the studies done in other geographical areas.

\begin{tabular}{|c|c|c|}
\hline Anti-TPO Positivity & Count & Percent \\
\hline Positive & 58 & 96.7 \\
\hline Negative & 2 & 3.3 \\
\hline
\end{tabular}

Table 3. Percentage Distribution of Thyroiditis According to Anti-TPO Positivity

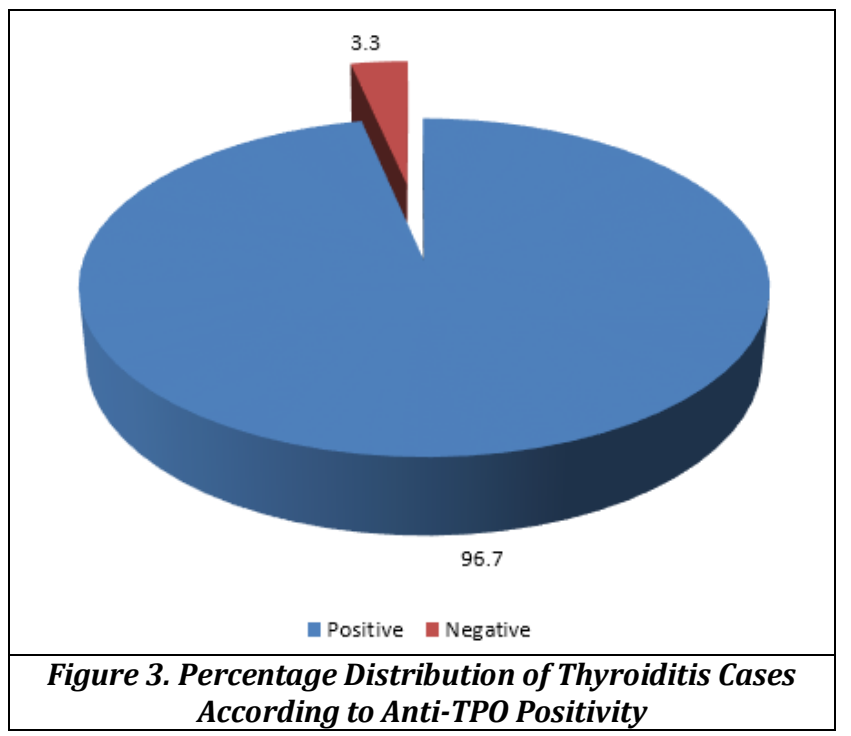

Percentage Distribution of Thyroiditis According to Thyroid Function

We know that Hashimoto's thyroiditis is one of the leading causes of hypothyroidism. And the incidence of hypothyroidism in Hashimoto's thyroiditis was 25\%, less than that of previous studies. $70 \%$ of patients were euthyroid and about 5\% were hyperthyroid irrespective of anti-TPO positivity.

\begin{tabular}{|c|c|c|}
\hline Thyroid function & Count & Percent \\
\hline Hypothyroidism & 15 & 25.0 \\
\hline Hyperthyroid & 3 & 5.0 \\
\hline Euthyroid & 42 & 70.0 \\
\hline Table 4. Percentage Distribution of Thyroiditis According \\
to Thyroid Function \\
\hline
\end{tabular}

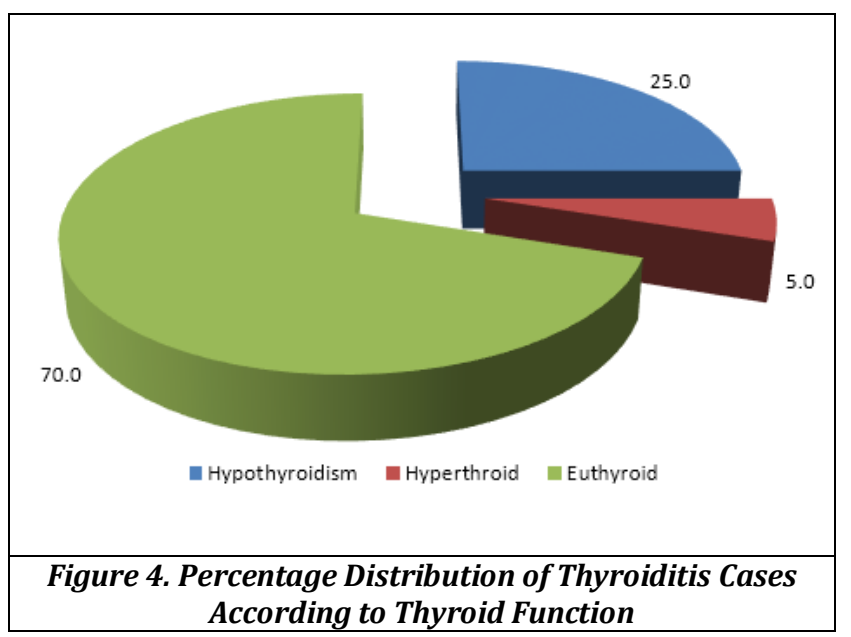

Association of Anti-TPO Positivity with Variables

When antithyroid positivity was compared with the percentage of hypothyroid, hyperthyroid and euthyroid, out of 60,14 were hypothyroid and antithyroid antibody positive which comes roughly about $93.3 \%$ of hypothyroid cases with FNAC proven thyroiditis were tested positive for antithyroperoxidase antibody. And about 41 out of 60 patients were euthyroid and $97 \%$ of the euthyroid cases were positive for antithyroid antibody. Only 3 were hyperthyroid and all of them tested positive for antithyroid antibody but were FNAC proven cases of thyroiditis. 


\begin{tabular}{|c|c|c|c|c|c|c|c|c|}
\hline \multicolumn{5}{|c|}{ Anti-TPO|Hypothyroidism|Hyperthyroid } & \multicolumn{2}{|c|}{ Euthyroid } & \multirow[b]{2}{*}{$\chi^{2}$} & \multirow[b]{2}{*}{$\mathbf{p}$} \\
\hline positivity & Count & $\%$ & Count & $\%$ & Count & $\%$ & & \\
\hline Positive & 14 & 93.3 & 3 & 100.0 & 41 & 97.6 & & \\
\hline Negative & 1 & 6.7 & 0 & 0.0 & 1 & 2.4 & & \\
\hline
\end{tabular}
Thyroid Function

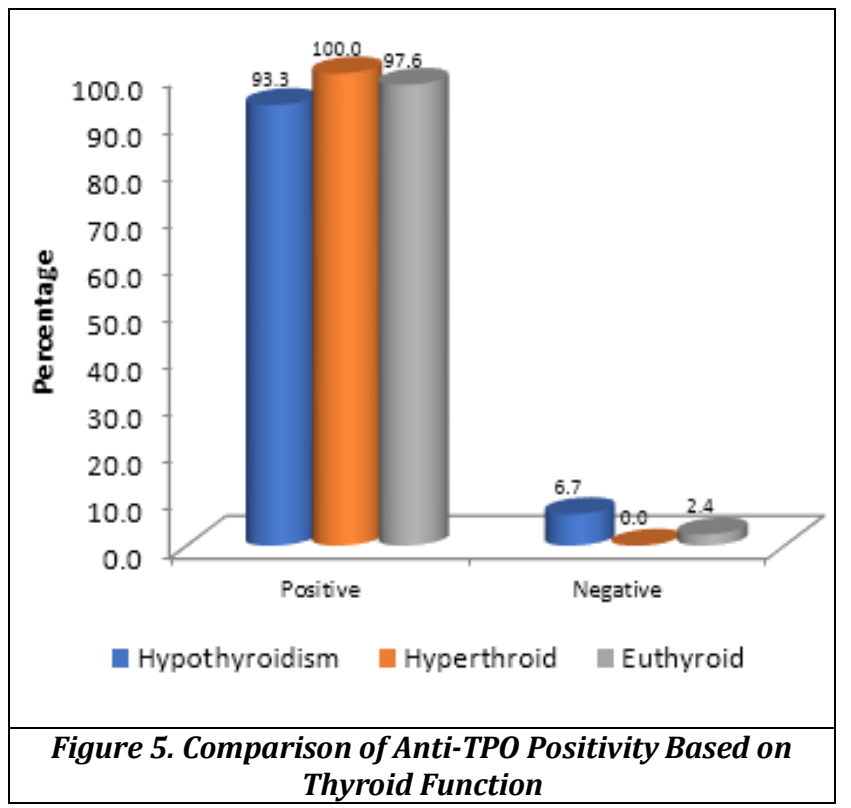

Age Wise Distribution of Antithyroid Antibody Positive Cases

The age group was divided as above 40 and below 40 . Among positive cases, 26 were below $40 y$ rs and 32 were above 40 yrs. $96 \%$ of below 40 were positive for anti thyroperoxidase antibody and $97 \%$ of above 40 were positive for antithyroperoxidase antibody.

\begin{tabular}{|c|c|c|c|c|c|c|}
\hline Anti-TPO & \multicolumn{2}{|c|}{$<=\mathbf{4 0}$} & \multicolumn{2}{c|}{$>\mathbf{4 0}$} & \multirow{2}{*}{$\mathbf{z}$} \\
\cline { 2 - 5 } Positivity & Count & $\mathbf{\%}$ & Count & $\mathbf{\%}$ & & \\
\hline Positive & 26 & 96.3 & 32 & 97.0 & \multirow{2}{*}{0.02} & \multirow{2}{*}{0.885} \\
\hline Negative & 1 & 3.7 & 1 & 3.0 & & \\
\hline \multicolumn{7}{|c|}{ Table 6. Comparison of Anti-TPO Positivity Based on Age } \\
\hline
\end{tabular}

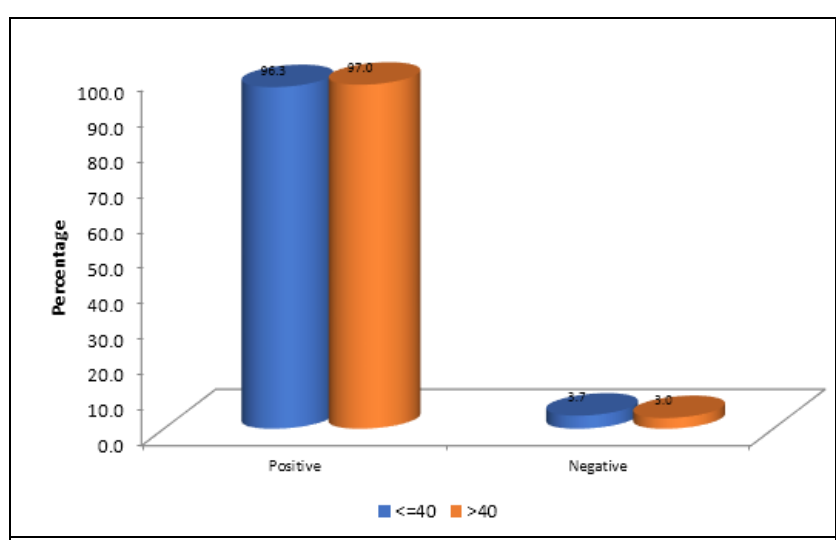

Figure 6. Comparison of Anti-TPO Positivity Based on Age

Comparison of Anti-TPO Positivity Based on Sex

Among the male thyroiditis patients all were found to be antiTPO antibody positive and among females, $96 \%$ were found to be anti-TPO antibody positive. There is definite female preponderance in number of antithyroid antibody positive cases.

\begin{tabular}{|c|c|c|c|c|c|c|}
\hline \multirow{2}{*}{$\begin{array}{l}\text { Anti-TPO } \\
\text { positivity }\end{array}$} & \multirow{2}{*}{\multicolumn{2}{|c|}{\begin{tabular}{c|} 
Male \\
Count $/$ Percent
\end{tabular}}} & \multirow{2}{*}{\multicolumn{2}{|c|}{\begin{tabular}{|c|} 
Female \\
Count $\mid$ Percent \\
\end{tabular}}} & \multirow[b]{2}{*}{$\chi^{2}$} & \multirow[b]{2}{*}{ p } \\
\hline & & & & & & \\
\hline Positive & 10 & 100.0 & 48 & 96.0 & \multirow{2}{*}{0.41} & \multirow{2}{*}{0.520} \\
\hline Negative & 0 & 0.0 & 2 & 4.0 & & \\
\hline
\end{tabular}

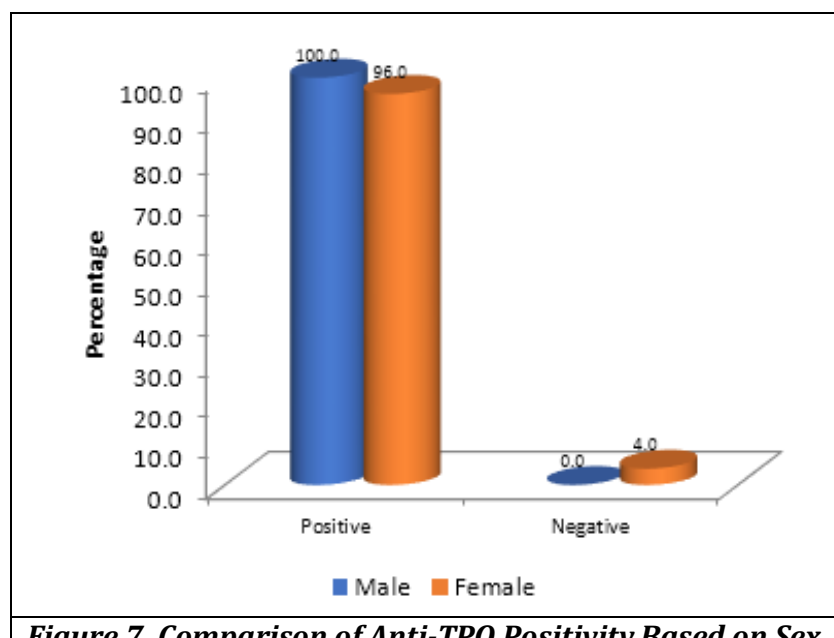

Figure 7. Comparison of Anti-TPO Positivity Based on Sex

\section{Association of Thyroiditis and Different Variables}

The age group was divided into below $40 \mathrm{yrs}$ and above $40 \mathrm{yrs}$. And the prevalence of hypothyroidism was almost same for both age groups with a slight increase of hypothyroidism in below 40 age group and prevalence of hyperthyroidism in above 40 age group.

\begin{tabular}{|c|c|c|c|c|c|c|c|c|}
\hline \multirow{2}{*}{ Age } & \multicolumn{2}{|c|}{ Hypothyroidism } & \multicolumn{2}{|c|}{ Hyperthyroid } & \multicolumn{2}{|c|}{ Euthyroid } & \multirow[b]{2}{*}{$\chi^{2}$} & \\
\hline & Count & $\%$ & Count & $\%$ & Count & $\%$ & & \\
\hline$<=40$ & 8 & 29.6 & 0 & 0.0 & 19 & 70.4 & & \\
\hline$>40$ & 7 & 21.2 & 3 & 9.1 & 23 & 69.7 & & \\
\hline
\end{tabular}

Table 8. Comparison of Age Based on Thyroid Function

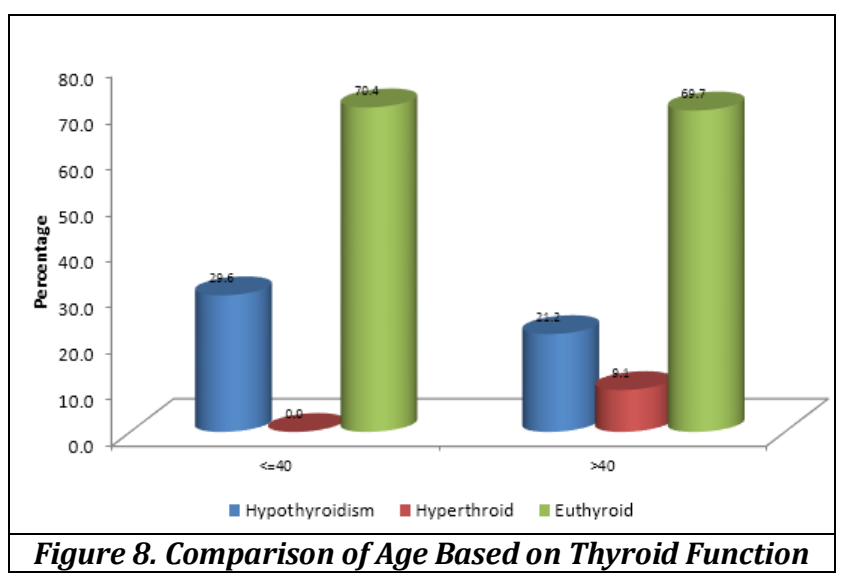

Comparison According to Sex in Relation to Thyroid Function in Thyroiditis Cases

$40 \%$ of male thyroiditis cases were hypothyroid and $22 \%$ of female thyroiditis cases were hypothyroid. Only $6 \%$ of female thyroiditis cases were hyperthyroid. $60 \%$ of male thyroiditis cases were euthyroid and $72 \%$ female thyroiditis cases were euthyroid. Since thyroiditis can be presented in any stage from hypothyroid to euthyroid to hyperthyroid, we cannot comment based on single visit observations and it needs follow up. 


\begin{tabular}{|c|c|c|c|c|c|c|c|c|}
\hline \multirow{2}{*}{ Sex } & Hypothyroid & Hyperthyroid & Euthyroid & \multirow{2}{*}{$\chi^{2}$} & $\mathbf{p}$ \\
\cline { 2 - 8 } & Count & $\mathbf{\%}$ & Count & $\mathbf{\%}$ & Count & $\mathbf{\%}$ & & \\
\hline Male & 4 & 40.0 & 0 & 0.0 & 6 & 60.0 & \multirow{2}{*}{1.85} & 0.396 \\
\hline Female & 11 & 22.0 & 3 & 6.0 & 36 & 72.0 & & \\
\hline Table 9. Comparison According to Sex Based on Thyroid \\
Function \\
\hline
\end{tabular}

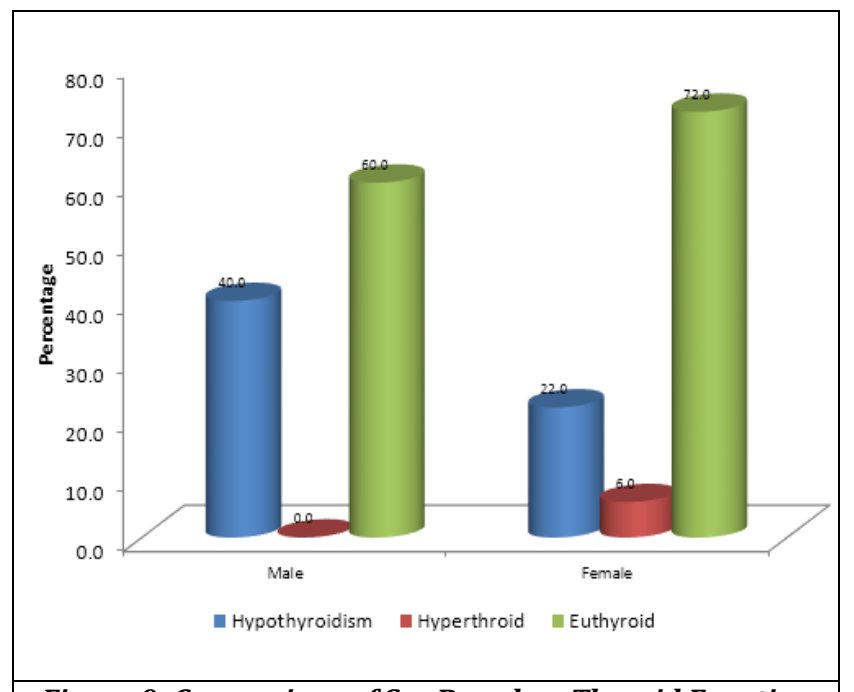

Figure 9. Comparison of Sex Based on Thyroid Function

\section{DISCUSSION}

Thyroid disorders are very common and increasing in incidence in the present scenario. Among all the disorders, one of the common problems is Hypothyroidism. And a leading cause of Hypothyroidism is Hashimoto's thyroiditis and it is usually diagnosed by ultrasonography and FNAC. Anti-TPO antibodies are used but its sensitivity and specificity is doubtful. Can we rely on anti-TPO antibody positivity itself to diagnose Hashimoto and not rely on FNAC alone? And the \% of anti-TPO antibody positivity has not been studied in this population. Here we tried to relate the presence of hypothyroidism with anti-TPO antibody positivity. And obtained certain results which tally with studies of incidence conducted on other populations.

The area of choice for the study was T D Medical College Alappuzha which is situated near to beach and draining area is mostly seaside. Here the rate of hypothyroidism and goitre is in alarming numbers as we get from OPD findings. Autoimmune thyroiditis cases also get confused with simple goitre and many Hashimoto thyroiditis patients undergo thyroidectomy. The problem with thyroidectomy for Hashimoto's is that thyroiditis cases usually have dense adhesions and chances of injuring recurrent laryngeal nerve and parathyroids is very high. And the morbidity for the patient is very high compared to management with nonoperative management.

Whether by doing the Anti-TPO antibody positivity, we can diagnose Hashimoto's and hence prevent surgery for such patients who have thyroiditis in FNAC. Currently Anti-TPO antibody testing is not part of workup of thyroid cases in surgery. Can we avoid unnecessary thyroid surgeries by including this test as a routine test?

From the analysis, we found that $>97 \%$ of cases of thyroiditis were anti-TPO antibody positive and among the 60 cases studied, only one was tested negative. So we can assume with $97 \%$ certainty that all the anti-TPO positives are
Hashimoto's thyroiditis and can be managed with drugs alone. Further it was found that, 30\% of thyroiditis cases were hypothyroid which is significant. And only 3 cases were hyperthyroid, and majority were euthyroid.

Pertaining to the disease course for autoimmune thyroiditis a person may go through all phases that is, euthyroid, hypothyroid and hyperthyroid hence we cannot comment that the $60 \%$ of the cases are euthyroid as the patient may develop hyper or hypothyroidism at any particular time.

Based on the demographic details obtained, females had more incidence than males of about $83 \%$ compared to $16 \%$ in males.

The age group with more case bulk was the 40 to 60 age group. And in males and females, the age group was comparable.

The primary objective of determining anti-TPO antibody positivity showed that $97.6 \%$ cases of FNAC proven thyroiditis cases to be positive. So, it can to a certain extent prove a case of autoimmune thyroiditis without imaging or FNAC.

Here, my study also tried to compare the hypothyroid, hyperthyroid and euthyroid patients among thyroiditis proven patients and $30 \%$ of them were found to be hypothyroid. And only one was hyperthyroid and rest were euthyroid. Among them, 93\% of hypothyroid, 100\% of hyperthyroid and $97 \%$ of euthyroid were anti-TPO antibody positive. $97 \%$ of above 40 age group and $93 \%$ of below 40 age group were anti-TPO antibody positive. $100 \%$ of males and $96 \%$ of female thyroiditis cases were anti-TPO antibody positive. $40 \%$ males were hypothyroid and $20 \%$ of females were hypothyroid.

\section{CONCLUSION}

Our study was done on FNAC proven cases of thyroiditis. We looked for antithyroid antibody positivity in these patients. It was an observational study and the idea was to see the accuracy with which the FNAC findings of thyroiditis correlated with anti-TPO antibody positivity. We also looked for thyroid function status of all the patients. Comparison was done in each group in relation to thyroiditis and anti-TPO antibody positivity. And the conclusions are-

1. $97 \%$ thyroiditis cases were positive for anti-TPO antibody.

2. $30 \%$ were hypothyroid cases, one case was hyperthyroid and rest euthyroid among thyroiditis cases.

3. The test for anti-TPO antibody positivity can be used to detect cases of Hashimoto's thyroiditis with 97\% certainty.

4. The anti-TPO antibody testing can be used along with regular testing of TFT, FNAC in cases of thyroiditis.

5. The functional status of thyroid cannot be defined based on thyroiditis as it may be in a different functional state at a particular time.

\section{REFERENCES}

[1] Hamburger JI. The various presentations of thyroiditis. Diagnostic considerations. Ann Intern Med 1986;104(2):219-24. 
[2] Müssig K, Künle A, Säuberlich AL, et al. Thyroid peroxidase antibody positivity is associated with symptomatic distress in patients with Hashimoto's thyroiditis. Brain Behav Immun 2012;26(4):559-63.

[3] Chandanwale SS, Gore CR, Bamanikar SA, et al. Cytomorphologic spectrum of Hashimoto's thyroiditis and its clinical correlation: a retrospective study of 52 patients. Cytojournal 2014;11:9.
[4] Unnikrishnan AG, Kalra S, Sahay RK, et al. Prevalence of hypothyroidism in adults: an epidemiological study in eight cities of India. Indian J Endocrinol Metab 2013;17(4):647-52.

[5] Unnikrishnan AG, Menon UV. Thyroid disorders in India: an epidemiological perspective. Indian J Endocrinol Metab 2011;15(Suppl 2):S78-S81. 